\title{
Angiogenesis in the Septum and Inner Membrane of Refractory Chronic Subdural Hematomas: Consideration of Findings after Middle Meningeal Artery Embolization with Low-concentration n-butyl-2-cyanoacrylate
}

\author{
Hiroshi Saito, ${ }^{1}$ Michihiro Tanaka, ${ }^{1}$ and Hiromu Hadeishi ${ }^{1}$
}

The middle meningeal artery (MMA) is suggested to play an important role in the recurrence of chronic subdural hematomas $(\mathrm{CSDH})$. However, the exact mechanisms involved in the recurrence of CSDHs still remain unknown. For recurring CSDHs, MMA embolization is performed using low-concentration $n$-butyl-2-cyanoacrylate (NBCA) at our hospital. We report new findings and a discussion related to the mechanism of CSDH recurrence based on the imaging findings after MMA embolization, and cases that required craniotomy due to recurrence. The study included eight patients with recurrent $\mathrm{CSDH}$, defined as ipsilateral hematoma re-enlargement within 3 months, and treated with MMA embolization. MMA embolization was performed successfully in all eight patients with no complications. Of the eight patients treated, one patient required craniotomy for the evacuation of CSDHs due to hematoma re-enlargement. NBCA casts were observed in the inner membrane of CSDHs in five of the eight patients by postoperative computed tomography scans. In the case that required craniotomy, the formation of neovasculatures was observed in the inner membrane of the CSDH. Our results suggested that angiogenesis also occurs in the septum and inner membrane through the MMA and the outer membrane of the CSDH. Additionally, these neovascular vessels may be involved in the recurrence of CSDH after MMA embolization. In a future larger study, it is necessary to elucidate in detail the vascular architecture of the CSDH membrane associated with the hematoma re-enlargement, and the effectiveness of MMA embolization that embolized to these peripheral neovascular vessels.

Keywords: chronic subdural hematoma, recurrence, embolization, angiogenesis, $n$-butyl-2-cyanoacrylate (NBCA)

${ }^{1}$ Department of Surgical Neurology, Kameda Medical Center, Kamogawa, Chiba, Japan

Received: November 10, 2018; Accepted: March 25, 2019

Online September 14,2019

Copyright $\odot 2019$ by The Japan Neurosurgical Society This work is licensed under a Creative Commons AttributionNonCommercial-NoDerivatives International License.

\section{Introduction}

Chronic subdural hematomas (CSDHs) are often found in the elderly peoples. It is expected that the number of people affected by this disease will increase as population aging increases. Although burr-hole surgery has been established as a standard treatment for CSDHs, recurrence rates of 5-33\% have been reported. ${ }^{1-7)}$ For intractable CSDH with repeated recurrence, the effectiveness of Ommaya reservoir placement,${ }^{1,8}$ subdural-peritoneal shunts, ${ }^{1,9)}$ the removal of the outer membrane of the hematoma capsule by craniotomy, ${ }^{1)}$ endoscopic septal perforation, ${ }^{10,11)}$ have been reported with treatment effects of a certain degree. However, they are not necessarily minimally invasive.

In CSDHs, the middle meningeal artery (MMA) and neovascular vessels in the outer membrane anastomose via the dura matter. This leads to the intermittent bleeding of vulnerable blood vessels and contributes to CSDH enlargement. ${ }^{12,13)}$ It is, therefore, believed that the embolization of the MMA can reduce the recurrence rate of CSDHs by disrupting blood flow to the outer membrane of hematomas. Although a few cases of recurrence after MMA embolization have been reported, much remains unknown about the mechanisms involved in the recurrence of CSDHs.

In addition to burr-hole surgery, we have been performing MMA embolization mostly using low-concentration $n$-butyl2-cyanoacrylate (NBCA) for recurring CSDHs at our hospital. Here, we discuss and report the mechanisms of hematoma enlargement derived from the findings of postembolization imaging tests, and cases that required craniotomy after embolization due to recurrence.

\section{Patients and Methods}

From June 2006 to October 2017, 714 patients with CSDHs underwent single burr-hole surgery with irrigation and drainage under local anesthesia in our hospital. For hard or multicellular hematomas, a drainage tube was inserted after the hematoma cavity was washed with artificial cerebrospinal fluid or normal saline. Subsequently, we performed MMA embolization on cases with two or more ipsilateral recurrences within 3 months and cases with high risks of recurrence in which antithrombotic/anticoagulant therapy could not be discontinued. Embolization was performed after sufficient explanations were provided and informed 
consent was obtained. Embolization was not performed if informed consent could not be obtained, renal dysfunction was present, use of the chemotherapy drugs affecting coagulation ability could be discontinued, there was difficulty in staying calm during embolization due to restlessness, and recurrence after more than 3 months of undergoing a burrhole surgery. After embolization, head computed tomographies (CTs) were performed on all patients immediately after surgery or a day after surgery to assess the penetration of the embolic material (NBCA).

\section{MMA Embolization}

Endovascular embolization was performed under local anesthesia using a biplane angio-graphic system (Philips Allura Clarity FD; Philips Healthcare, Andover, MA, USA). A 5- or 6-Fr long sheath was placed in the right femoral or brachial artery, and a 5- or 6-Fr guiding catheter was inserted into the external carotid artery using standard coaxial techniques. For microcatheters (MCs), a 1.5-Fr Baltacci (Balt Extrusion INC., Montmorency, France) was used. The MC was introduced to the periphery of the MMA not involved with dangerous anastomoses to perform superselective angiography and observe abnormal vascular networks. Embolization was performed, using NBCA (Braun, Melsungen, Germany) as an embolic material, while paying attention to dangerous anastomoses defined as recurrent meningeal artery, the feeding vessel to the facial nerve, or the connection with the internal carotid artery through the inferolateral trunk, and after confirming that there was no involvement of these dangerous anastomoses. The NBCA was diluted to a concentration of $12-24 \%$ using sufficiently warmed Lipiodol (Guerbet LLC., Bloomington, IN, USA). The embolization was performed by extremely slow injection of low concentration NBCA over a long period of time, to ensure the cast reached the periphery of the MMA (Fig. 1). In one of the eight cases, polyvinyl alcohol (PVA; Boston Scientific, MA, USA) particles (size: $50-150 \mu \mathrm{m}$ ) were used in combination with NBCA for treatment.

\section{Results}

In 84 cases $(11.8 \%)$, there was a first recurrence of CSDHs, while there was a second recurrence in 18 cases $(2.5 \%)$. In MMA embolization was performed on eight patients with intractable CSDHs that recurred twice or more within 3 months (Table 1).

The patients that underwent MMA embolization were all men with a mean age of 79 years (64-87 years) and an average postoperative observation period of 28.9 months (Table 1). Two of the eight patients were taking oral antiplatelets or anticoagulants. In seven cases, except for one patient with carotid artery stenosis, there was no abnormal vascular network detected on the CSDH's membrane on internal carotid artery angiography. The abnormal vascular networks were observed in all cases on the selective MMA angiography before embolization and confirmed to have disappeared after embolization. No complications associated with embolization were observed in any of the patients, and there was no recurrence of CSDHs in seven of the eight
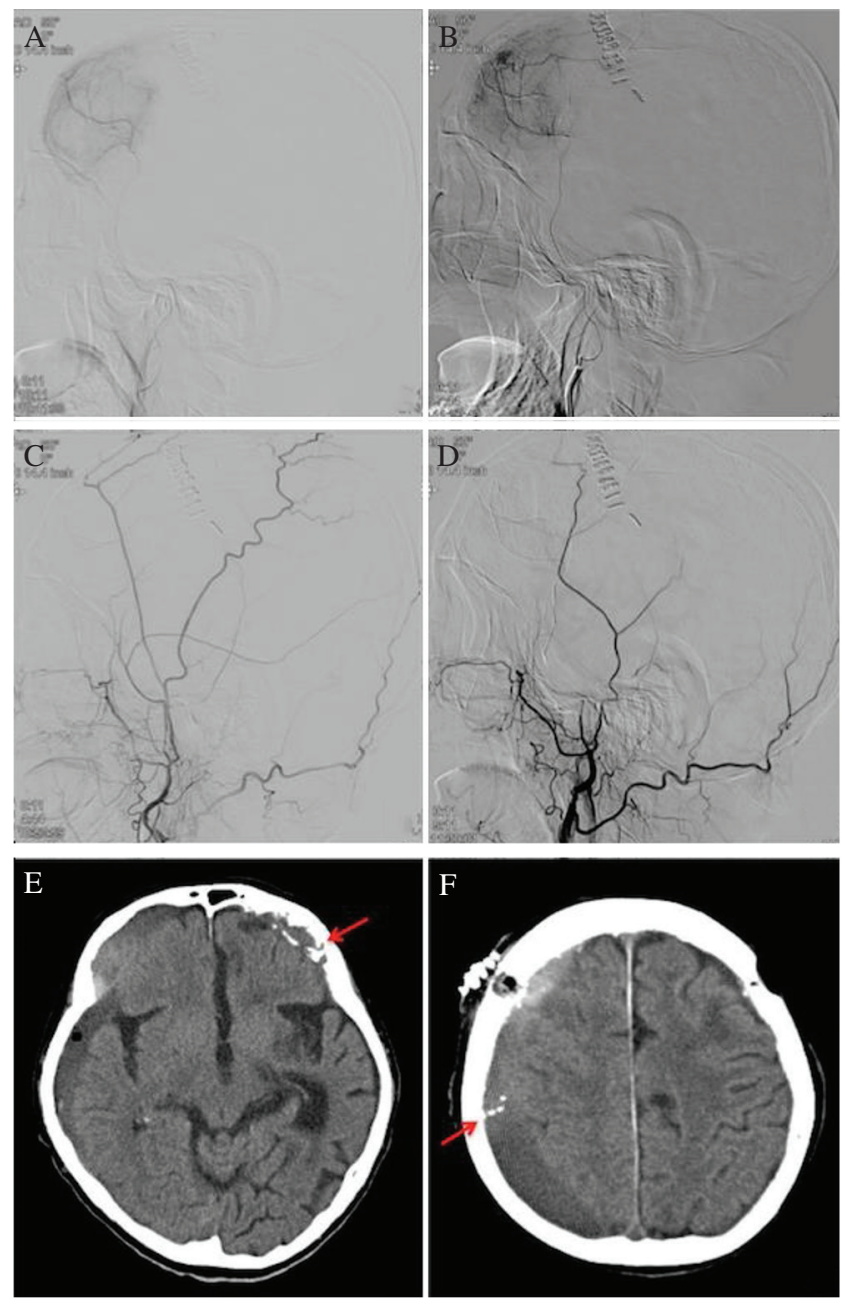

Fig. 1 A lateral pre-operative superselective angiogram of the right (A) and left (B). Abnormal vascular networks were observed within the bilateral middle meningeal artery's frontal branch. Each artery was embolized using 17\% n-butyl-2-cyanoacrylate (NBCA). A postprocedural digital subtraction angiogram showed that the abnormal vascular networks disappeared (C and D). Post-embolization computed tomography scans revealed the NBCA cast (arrows) in the inner membrane and septum of the chronic subdural hematoma (E and F).

patients after embolization. In one of the eight patients, hematoma size was reduced after embolization, and did not require burr-hole surgery. Plain CTs of the head, performed immediately after embolization or 1 day after embolization, showed no aberrations of the NBCA cast in the intracranial blood vessels. We observed that the NBCA cast had reached, not only the outer membrane of the hematoma capsule, but also the inner membrane via the septum and the periphery of the hematoma in five of the eight patients (Figs. 1E, 1F, 2C and 2D). One patient presented recurrence 3 months after the MMA embolization through the right brachial artery (Fig. 2). The patient underwent Ommaya reservoir placement surgery, but he also required craniotomy for hematoma removal 13 months after embolization because of recurrence due to occlusion of the Ommaya reservoir. Intraoperative findings showed the formation of neovascular vessels in the inner and outer membrane of the hematoma capsule (Fig. 3). 


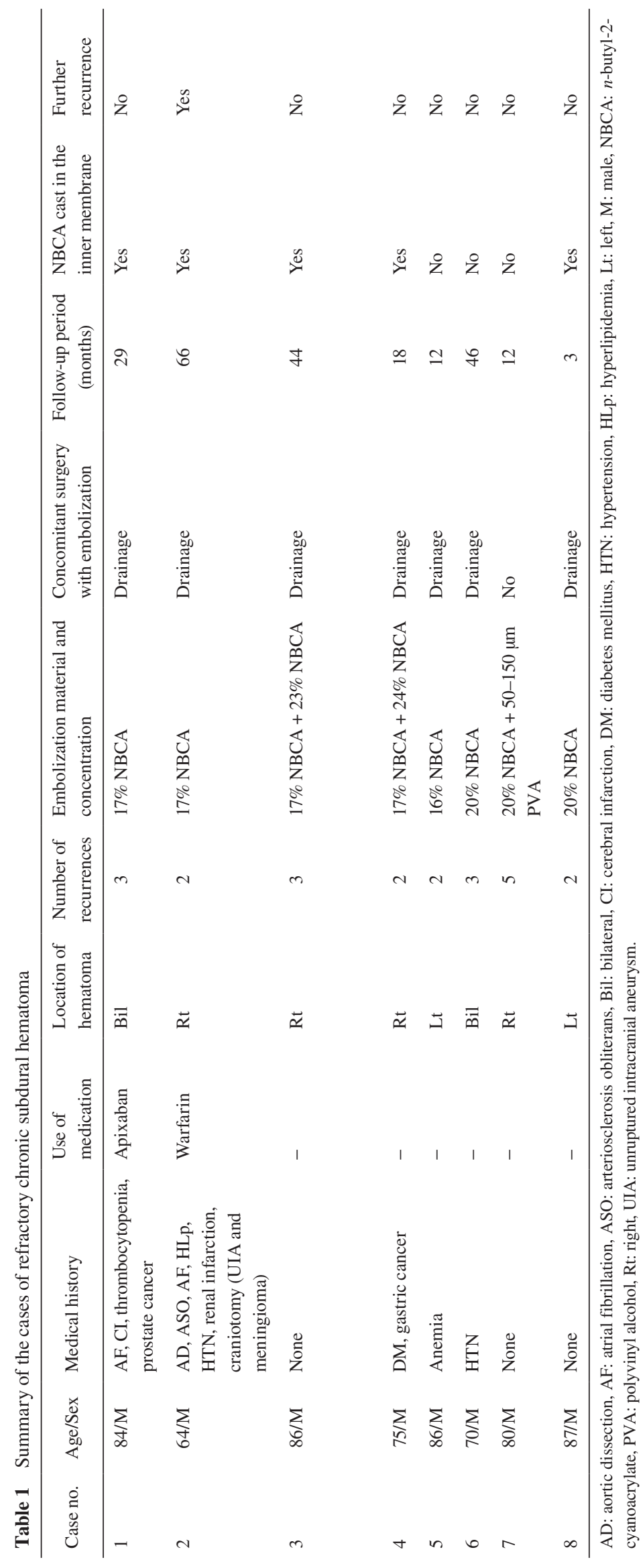




\section{Discussion}

Middle meningeal artery embolization for recurrent CSDHs was first reported by Mandai et al. ${ }^{14)}$ There have been numerous reports on its effectiveness ever since. ${ }^{14-23)}$ Ban et al. ${ }^{15)}$ performed MMA embolization on 72 patients and reported positive treatment outcomes with no surgical complications and only one case of recurrence. The MMA has latent anastomoses at the ophthalmic artery, the internal carotid artery and the cranial nerves. MMA embolization is
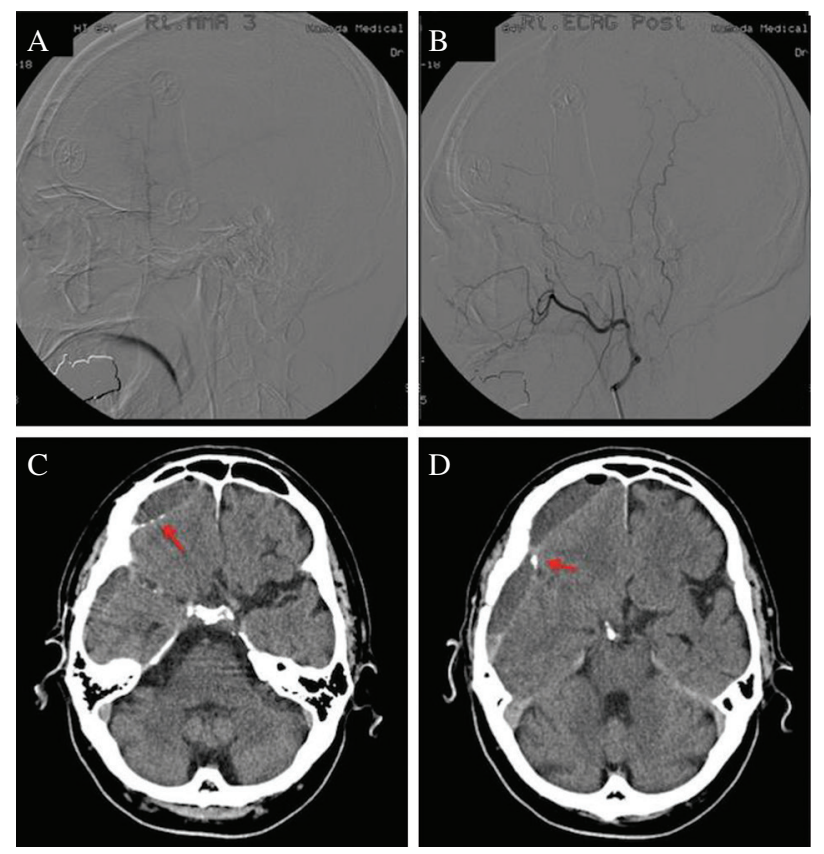

Fig. 2 Superselective angiographies of the right middle meningeal artery (MMA) obtained before (A) and after (B) embolization in Case 2 with $17 \%$-butyl-2-cyanoacrylate (NBCA). The MMA is completely embolized and the abnormal vascular networks are no longer seen. Post-embolization computed tomography scans revealed the NBCA cast (arrows) in the inner membrane and septum of the chronic subdural hematoma (C and D). considered to be a minimally invasive and safe treatment if attention is paid to these locations.

In CSDHs, a hematoma cavity is formed as a result of a ruptured dural border cell layer due to minor injuries that lead to an influx of spinal fluid and blood inside. Histopathologically, hematoma capsules have a layered structure of two to three layers, while there are numerous capillary-like vessels, venules and arterioles in the outer membrane which anastomose with the dura mater via the MMA. ${ }^{12,24)}$ These neovascular vessels are observed as characteristic dark contrast images of foggy staining, cotton wool-like staining, and an abnormal vascular network in a superselective angiography of the MMA. ${ }^{16,18)}$ Intermittent bleeding into the hematoma cavity caused by the ruptured neovascular vessels present in the outer membrane is considered to be a factor that leads to the enlargement of CSDHs. On the other hand, much remains unclear with regard to the involvement of the inner membrane of hematomas. Yamashima et al. ${ }^{25)}$ reported that the inner membrane of hematoma capsules form syncytia that reinforce the arachnoid membrane and, unlike in the outer membrane, neovascularization does not take place in them. However, studies that observed hematoma cavities during surgery under neuroendoscopy reported bleeding from the columnar structure (septum) present in the cavities in addition to bleeding from the outer membrane of hematomas, and that this may have been involved in hematoma enlargement. ${ }^{11,26)}$ Nakaguchi et al. ${ }^{27)}$ also focused on the contrast effect of the inner membrane of hematomas in contrast $\mathrm{CT}$ and surmised that there was the development of neovascular vessels in the inner membrane as well. Furthermore, they also suggested the involvement of angiogenesis in the inner membrane in the enlargement of the hematoma, because the contrast effect of the inner membrane increased as the chronic phase of a hematoma progressed. In five of the eight cases that we analyzed, we observed an influx of NBCA in the inner membrane of hematomas from the columnar structure and the periphery of the hematomas on $\mathrm{CT}$ scans performed after embolization. In the case where
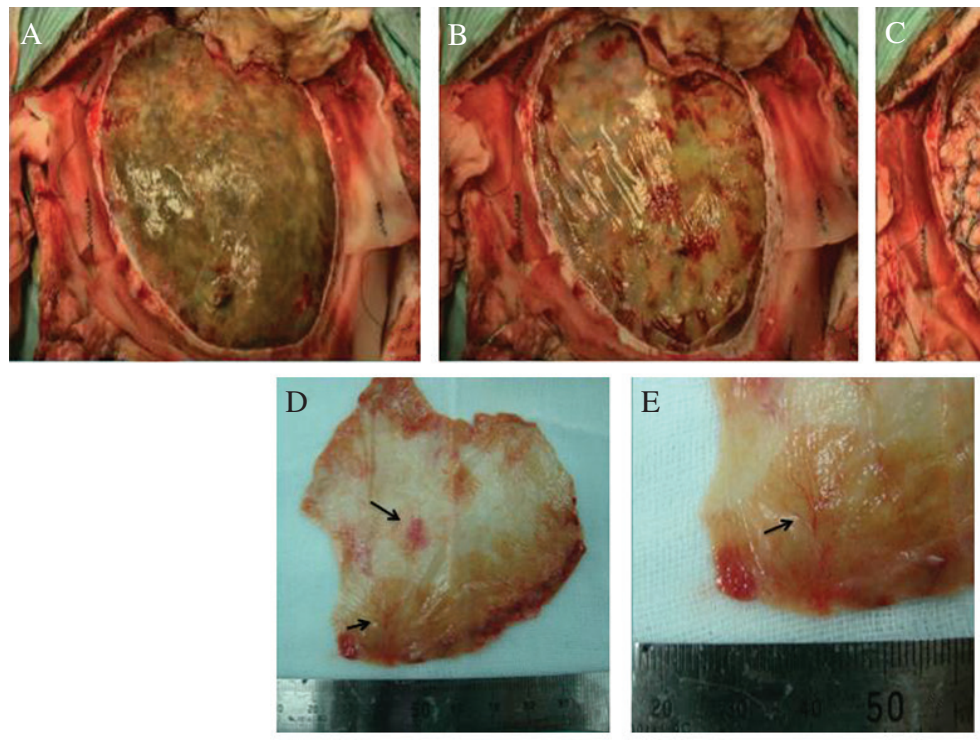

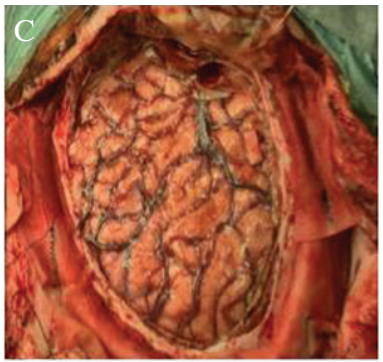

Fig. 3 The craniotomy findings (Case 2). The outer membrane (A) and inner membranes (B) of the chronic subdural hematoma (CSDH). Post-resection of the inner membrane of the CSDH (C). The formation of neovasculatures (arrows) observed in the inner membrane of the CSDH (D and E). 
craniotomy was required due to re-enlargement, the formation of neovascular vessels was noted in the inner membrane of the hematoma (Figs. 3D and 3E). These findings suggest that vascular hyperplasia also occurs in the inner membrane and septum of hematomas, and these neovascular vessels may be involved in the recurrence of CSDH after MMA embolization (Figs. 1E, 1F, 2C and 2D). It remains unclear how neovascular vessels formed in the inner membrane and septum of hematomas affect the recurrence of CSDHs. However, it is assumed that cell proliferation, including the formation of capsules and enlargement of hematomas, and increased local metabolism take place in areas other than the outer membrane of hematomas. Moreover, there are cases in which CSDHs recurred even after the removal of hematomas or the outer membrane of hematomas via craniotomy. ${ }^{28)}$ In addition, MMA embolization is believed to block oxygen and nutrition supply at the inner membrane level of hematomas, which are required, not only for the outer membrane of hematomas, but also for $\mathrm{CSDH}$ enlargement, thereby greatly reducing the risk of recurrence. Much remains unknown about the vascular architecture involved in the recurrence of CSDHs. Therefore, further research is required.

The use of embolic materials such as NBCA, PVA and coils/gelatin sponges for MMA embolization have been reported. ${ }^{14-23)}$ Previous studies have also reported that the recurrence of CSDHs after MMA embolization is very less, ${ }^{14-23)}$ including the present case. Takeshima et al. ${ }^{29)}$ reported a case with recurrence after embolization with gelatin sponges alone, MMA recanalization occurred, and the mechanism was thought to have involved resorption of the gelatin sponge. Chihara et al. reported a case in which craniotomy was performed due to recurring CSDHs that happened 3 months after MMA embolization. They indicated the possibility that the development of collateral circulation to the outer membrane of the hematoma before CSDH healing may have been the cause. ${ }^{30)}$ To prevent recanalization, we used NBCA because it lowers the incidence of reopening after embolization. ${ }^{31)}$ NBCA is a cyanoacrylate-type adhesive that has the property of polymerizing upon contact with anions in the blood, hardening, and adhering to the vessel wall and MC tip. The lower the NBCA concentration, the lower the viscosity within the catheter, and the average polymerization time is extended to $3.2 \mathrm{~s}$ at $50 \%$ and to $11.8 \mathrm{~s}$ at $20 \%$. Additionally, visibility at the time of embolization increases by adding more Lipiodol as an oily contrast medium. As a result of reducing the concentration, adhesion to the catheter decreases and polymerization time is prolonged, thereby enabling infusion for a long period of time. ${ }^{32,33)}$ On the other hand, the viscosity of Lipiodol is $27-54 \mathrm{~mm}^{2} / \mathrm{s}$ at $20^{\circ} \mathrm{C}$, which is more than 10 times higher than that of non-ionic contrast medium used for cerebral angiography. ${ }^{34)}$ Thus, simply decreasing the NBCA concentration reduces the viscosity, and even if NBCA is injected for a long time, the cast often does not flow significantly into the periphery. Since Lipiodol has the characteristic of having lower viscosity after warming, it is suggested that Lipiodol be sufficiently warmed prior to use to prevent proximal occlusion. Due to the involvement of various factors such as the position of the
MC tip and speed of the NBCA injection performed by an operator, how far toward the peripheral region of the hematoma capsule the NBCA reaches by decreasing its concentration depends on the case. In our experience, when a flow-dependent $\mathrm{MC}$ was inserted as far as possible into the peripheral region of the MMA and 12-24\% NBCA was injected, the NBCA cast could be injected into the columnar structure or inner membrane of the CSDH in five of eight cases. In the past, the use of low-concentration NBCA in the preoperative embolization of meningioma was also reported to allow NBCA to reach the peripheral vascular bed in the tumor. ${ }^{32)}$ Based on the hypothesis that the formation of neovascular vessels in the inner membrane of hematomas is a cause of CSDH re-enlargement, we believe penetrating the periphery with low-concentration NBCA mixed with warm Lipiodol is effective for treatment. Moreover, NBCA is reported to present no difference in its effects for vascular occlusion at different concentrations; ${ }^{35)}$ thus, if NBCA can reach more peripheral neovascular vessels, potential blocking of the blood flow from the collateral circulation can be expected.

On the other hand, one of points to note in the use of lowconcentration NBCA is that, similar to the normal concentration of NBCA, there are risks of the rupture of the MC and vascular damage when the tip of a catheter is trapped after long-distance insertion. In addition, when removing a cast of low-concentration NBCA, one should pay attention to the scattering of NBCA to proximal vessels due to the softness of the cast. ${ }^{36)}$ To reduce these risks, Fukuda et al. ${ }^{32)}$ reported the importance of using a 4-Fr intermediate catheter and slowly removing a catheter after NBCA injection. No complications resulting from the use of low-concentration NBCA for recurrent CSDH has been reported in any study, ${ }^{16,18,20)}$ including ours. However, all reports are small-scale studies and thus, further investigation is needed.

The limitations of this study include the small number of cases and single-center, retrospective nature of the study. Further investigations with a larger number of cases are needed to confirm our results.

\section{Conclusion}

Middle meningeal artery embolization is expected to prevent CSDH recurrence with lower complication rates, provided that the understanding of the functional anatomy of the MMA is performed. Our results suggested that angiogenesis also occurs in the inner membrane and septum of hematomas through the MMA, and this neovascularization may be related to hematoma enlargement after MMA embolization. To perform more effective embolization, it is necessary to elucidate in detail the vascular architecture in the $\mathrm{CSDH}$ membrane in large study.

\section{Conflicts of Interest Disclosure}

All authors have no conflicts of interest to disclose.

\section{References}

1) Ducruet AF, Grobelny BT, Zacharia BE, et al.: The surgical management of chronic subdural hematoma. Neurosurg Rev 35: 155-169, 2012 
2) Liu W, Bakker NA, Groen RJ: Chronic subdural hematoma: a systematic review and meta-analysis of surgical procedures. J Neurosurg 121: 665-673, 2014

3) Mori K, Maeda M: Surgical treatment of chronic subdural hematoma in 500 consecutive cases: clinical characteristics, surgical outcome, complications, and recurrence rate. Neurol Med Chir (Tokyo) 41: 371-381, 2001

4) Santarius T, Kirkpatrick PJ, Ganesan D, et al.: Use of drains versus no drains after burr-hole evacuation of chronic subdural haematoma: a randomised controlled trial. Lancet 374: 1067-1073, 2009

5) Santarius T, Qureshi HU, Sivakumaran R, Kirkpatrick PJ, Kirollos RW, Hutchinson PJ: The role of external drains and peritoneal conduits in the treatment of recurrent chronic subdural hematoma. World Neurosurg 73: 747-750, 2010

6) Takeda N, Sasaki K, Oikawa A, Aoki N, Hori T: A new simple therapeutic method for chronic subdural hematoma without irrigation and drainage. Acta Neurochir (Wien) 148: 541-546, 2006

7) Weigel R, Schmiedek P, Krauss JK: Outcome of contemporary surgery for chronic subdural haematoma: evidence based review. J Neurol Neurosurg Psychiatry 74: 937-943, 2003

8) Sato M, Iwatsuki K, Akiyama C, Kumura E, Yoshimine T: Implantation of a reservoir for refractory chronic subdural hematoma. Neurosurgery 48: 1297-1301, 2001

9) Misra M, Salazar JL, Bloom DM: Subdural-peritoneal shunt: treatment for bilateral chronic subdural hematoma. Surg Neurol 46: 378-383, 1996

10) Hellwig D, Kuhn TJ, Bauer BL, List-Hellwig E: Endoscopic treatment of septated chronic subdural hematoma. Surg Neurol 45: 272-277, 1996

11) Shiomi N, Hashimoto N, Takeuchi H, Yamanaka T, Nakagawa N, Mineura K: [Endoscopic findings in chronic subdural hematoma]. No Shinkei Geka 30: 717-722, 2002 (Japanese)

12) Tanaka T, Kaimori M: [Histological study of vascular structure between the dura mater and the outer membrane in chronic subdural hematoma in an adult]. No Shinkei Geka 27: 431-436, 1999 (Japanese)

13) Takizawa K, Sorimachi T, Ishizaka H, et al.: Enlargement of the middle meningeal artery on MR angiography in chronic subdural hematoma. J Neurosurg 124: 1679-1683, 2016

14) Mandai S, Sakurai M, Matsumoto Y: Middle meningeal artery embolization for refractory chronic subdural hematoma. Case report. J Neurosurg 93: 686-688, 2000

15) Ban SP, Hwang G, Byoun HS, et al.: Middle meningeal artery embolization for chronic subdural hematoma. Radiology 286: 992-999, 2018

16) Hashimoto $T$, Ohashi $T$, Watanabe $D$, et al.: Usefulness of embolization of the middle meningeal artery for refractory chronic subdural hematomas. Surg Neurol Int 4: 104, 2013

17) Hirai S, Ono J, Odaki M, Serizawa T, Nagano O: Embolization of the middle meningeal artery for refractory chronic subdural haematoma. Usefulness for patients under anticoagulant therapy. Interv Neuroradiol 10(Suppl 2): 101-104, 2004

18) Ishihara $H$, Ishihara $S$, Kohyama $S$, et al.: Experience in endovascular treatment of recurrent chronic subdural hematoma. Interv Neuroradiol 13(Suppl 1): 141-144, 2007

19) Kim E: Embolization therapy for refractory hemorrhage in patients with chronic subdural hematomas. World Neurosurg 101: 520-527, 2017
20) Matsumoto $H$, Hanayama $H$, Okada $T$, et al.: Which surgical procedure is effective for refractory chronic subdural hematoma? Analysis of our surgical procedures and literature review. J Clin Neurosci 49: 40-47, 2018

21) Mino M, Nishimura S, Hori E, et al.: Efficacy of middle meningeal artery embolization in the treatment of refractory chronic subdural hematoma. Surg Neurol Int 1: 78, 2010

22) Takahashi K, Muraoka K, Sugiura T, et al.: [Middle meningeal artery embolization for refractory chronic subdural hematoma: 3 case reports]. No Shinkei Geka 30: 535-539, 2002 (Japanese)

23) Tempaku A, Yamauchi S, Ikeda H, et al.: Usefulness of interventional embolization of the middle meningeal artery for recurrent chronic subdural hematoma: five cases and a review of the literature. Interv Neuroradiol 21: 366-371, 2015

24) Nagahori T, Nishijima M, Takaku A: [Histological study of the outer membrane of chronic subdural hematoma: possible mechanism for expansion of hematoma cavity]. No Shinkei Geka 21: 697-701, 1993 (Japanese)

25) Yamashima T: The inner membrane of chronic subdural hematomas: pathology and pathophysiology. Neurosurg Clin N Am 11: 413-424, 2000

26) Matsuzawa M, Taguchi Y, Yamashita K, Miyakita Y, Sekino H: [Endoscopic findings of the chronic subdural hematoma cavities]. Shinkei Gaisho 20: 115-120, 1997 (Japanese)

27) Nakaguchi H, Yoshimasu N, Tanishima T: [Relationship between the natural history of chronic subdural hematoma and enhancement of the inner membrane on post-contrast CT scan]. No Shinkei Geka 31: 157-164, 2003 (Japanese)

28) Svien HJ, Gelety JE: On the surgical management of encapsulated subdural hematoma: a comparison of the results of membranectomy and simple evacuation. J Neurosurg 21: 172-177, 1964

29) Takeshima Y, Kawai S, Yonezawa T, Masui K, Nishi N: Middle meningeal artery embolization for intractable chronic subdural hematoma: a case report. Med J Osaka Gen Med Cen 27: 25-28, 2004 (Japanese)

30) Chihara H, Imamura H, Ogura T, Adachi H, Imai Y, Sakai N: Recurrence of a refractory chronic subdural hematoma after middle meningeal artery embolization that required craniotomy. NMC Case Rep J 1: 1-5, 2014

31) Natarajan SK, Born D, Ghodke B, Britz GW, Sekhar LN: Histopathological changes in brain arteriovenous malformations after embolization using Onyx or $n$-butyl cyanoacrylate: laboratory investigation. J Neurosurg 111: 105-113, 2009

32) Fukuda K, Higashi T, Okawa M, et al.: Preoperative embolization of meningioma with ultra-low concentration $n$-butyl 2-cyanoacrylate. J Neuroendovasc Ther 8: 259-265, 2014

33) Stoesslein F, Ditscherlein G, Romaniuk PA: Experimental studies on new liquid embolization mixtures (histoacryl-lipiodol, histoacrylpanthopaque). Cardiovasc Intervent Radiol 5: 264-267, 1982

34) Ayabe J, Kubo A, Maeda M, et al.: Usefulness of oily iodine contrast medium for embolization of meningioma. J Neuroendovasc Ther 10: $165-169,2016$

35) Sadato A, Wakhloo AK, Hopkins LN: Effects of a mixture of a low concentration of $n$-butylcyanoacrylate and ethiodol on tissue reactions and the permanence of arterial occlusion after embolization. Neurosurgery 47: 1197-1203; discussion 1204-1205, 2000

36) $n$-BCA Trail Investigators: $N$-butyl cyanoacrylate embolization of cerebral arteriovenous malformations: results of a prospective, randomized, multi-center trial. AJNR Am J Neuroradiol 23: 748-755, 2002

Corresponding author:

Hiroshi Saito, MD, PhD, Department of Surgical Neurology, Kameda Medical Center, 929 Higashi-cho, Kamogawa, Chiba 296-8602, Japan.

$\triangle$ saito.hiroshi.2@kameda.jp 